\title{
MEDAL AWARDS OF THE ROYAL SOCIETY*
}

\section{Copley Medal}

$\mathrm{T}$ HE Copley Medal has been awarded to Prof. Paul Langevin.

Prof. Langevin was one of the band of young pioneers who, in the closing years of the last century, were engaged in exploring the field which had been opened up by J. J. Thomson's discovery of the electron. He spent a year (1897-98) at the Cavendish Laboratory, and his Paris Dr. ès Sc. thesis (1902) is dedicated to J. J. Thomson. The thesis, a notable one, was entitled "Recherches sur les Gaz Ionisés". It dealt mainly with the recombination and mobilities of ions, their coefficients and the relations between them. It is a standard work on this subject. In it he also devised and applied new and elegant methods of measuring these quantities which were an advance on all their predecessors and have not since been improved upon to any appreciable extent. Related to this, and coming later, were important contributions to the theory of the diffusion of gaseous ions and its relation to ionic mobilities.

Langevin's greatest achievement is the foundation of the electron theory of magnetism. The theories of paramagnetism and of diamagnetism are still very much as he made them and left them more than thirty years ago.

There are few branches of contemporary physics which he has not illuminated and improved by his writings, and his work generally has the qualities of breadth, clearness, elegance and completeness which stamp the master.

$\mathrm{He}$ has had a great international influence. $\mathrm{He}$ has been a prominent figure at all the meetings of the conferences arranged by the Institut International de Physique Solvay since they started in 1911. On the death of Lorentz he was chosen to succeed him as president of the above. Institut.

He was awarded the Hughes Medal in 1915 and elected a Foreign Member of the Society in 1928.

[See NatuRE of November 30, p. 715.]

\section{RuMFord Medal}

The Rumford Medal has been awarded to Prof. Karl Manne Georg Siegbahn.

Prof. Siegbahn, member of an old Swedish family, is, in the field of X-rays, what Rowland was in the field of ordinary optics sixty years ago. He has introduced high precision into X-ray measurements. For example, the most accurate wave-length measurements in 1913 were those of Moseley, with an accuracy of about 1 per cent. By a brilliant succession of improvements in methods, design, inventions and technique, Siegbahn by 1924 had improved this to 0.001 per cent-a factor of 1,000 .

Siegbahn is -not only a great physicist, he is also a great engineer. He has made inventions and improvements in almost every useful type of apparatus connected with X-ray measurements, pumps, gratings, $\mathrm{X}$-ray tubes, ruling machines, etc.

Among his many achievements are the determination of the structure of the $L$ series of X-ray spectra, the discovery of the anomalous dispersion of X-rays * From the remarks made by Sir William Bragg in presenting the medals for 1940 . (with Hjalmar), the accurate and direct measurement of the grating spaces of calcite and rock salt, and the selection rules for the frequencies of X-ray absorption edges. In conjunction with Larsson and Waller he was the first to deviate X-rays with a prism. They developed this method until they could obtain by it measurements of refractive indexes for X-rays which are of quite surprising accuracy.

In recent years he has been much occupied with the development and improvement of methods of measurement of 'ultra-soft' X-rays, the region between about 10 and $500 \mathrm{~A}$., and with conspicuous success. This covers the gap between the ordinary $\mathrm{X}$-ray region and the optical region of radiation.

$\mathrm{He}$ and his students have also been much interested in the values of the fundamental constants of physics and have made important contributions to our knowledge of them.

H.e has written a masterly book on the spectroscopy of X-rays. An extraordinarily large proportion of the information in it is due to the work of himself and his students. He has created an outstanding school at Uppsala and Stockholm, which now represents most of the physics of Sweden.

$\mathrm{He}$ was awarded the Hughes Medal in 1934.

A Royal Medal has been awarded to Prof. Patrick Maynard Stuart BlacketT.

Prof. Blackett is especially distinguished for his work on cosmic rays and the particles connected with them.

The early work which first brought his name into prominence was concerned with the disintegration of nitrogen by $\alpha$-particles; arising out of the experimental observations was the convincing proof that the disintegration process originated in the actual capture of the $\alpha$-particle by the nitrogen nucleus.

The demonstration of cosmic-ray showers was one of Blackett's early successes with direct Wilson chamber photographs; but perhaps his most spectacular discovery-made simultaneously by Anderson in America--was that, in a large cloud chamber controlled by the tripping of counters, tracks appeared which could only be explained as due to a new particle-the positive electron. The importance of this discovery in the light of Dirac's theory was immediately realized by Blackett and his co-worker Occhialini, and important results have emerged.

Blackett also--in collaboration with Chadwick and Occhialini-extended the work on the positive electron, and it was soon found that there were sources other than cosmic rays. With the same collaboration Blackett was also instrumental in showing that quanta of sufficient energy could produce a pair of electrons, and this production was related to the so-called nuclear absorption of $\gamma$-rays-a phenomenon previously known but until then unexplained.

Blackett has followed up his cosmic-ray work and has published a number of very interesting papers dealing with various aspects of these rays. He has measured their energy, inferring therefrom a cosmicray energy spectrum; he has observed, with an extraordinarily high degree of accuracy, the scattering and energy loss of cosmic-ray particles in their 
passage through metal plates and has discussed the nature of the penetrating component of cosmic rays. Two papers on this topic appeared in the Proceedings of the Royal Society for 1938.

Lastly, reference must not be omitted of Blackett's important experimental contributions to our knowledge of the heavy electron - the particle which seems destined to be of such importance in the understanding of the more familiar nuclear particles.

A Royal Medal has been awarded to Dr. Francis Hugh Adam Marshali.

Dr. Marshall's earlier research work (1903-7) on the cestrous cycle, corpus luteum, and removal and grafting of ovaries laid the foundations for all the modern discoveries concerning the internal secretions of the sex organs.

The publication of his large text-book on the "Physiology of Reproduction", in 1910, stimulated work on this subject throughout the world, not only on points of scientific interest but also in regard to the application to medicine and to questions of fertility and milk secretion in the domestic animals. $\mathrm{He}$ was engaged on a third edition of this book when war broke out. He is generally acknowledged to be the father of this subject, and but few papers on this branch of science to-day are published without some reference to his work.

Arising out of his research, and that of his pupils, the importance of the anterior pituitary as a source of internal secretions, affecting not only the sex organs but also other body functions, has been recognized, and has led the way to an enormous volume of research work in recent years throughout the world. It is one of the subjects in which the greatest ad. vances have been made in recent years.

Recently, his research has been concentrated on the exteroceptive factors, such as light, ultra-violet irradiation and nerve stimuli, which affect the sex organs by way of their effects on the anterior pituitary. This work explains the physiological basis for the seasonal and other changes which occur in reproductive activity. He summarized this aspect of the subject in the 1936 Croonian Lecture on "Sexual Periodicity and the Causes which Determine It", besides adding new matter.

\section{Davy Medal}

The Davy Medal has been awarded to Prof. Harold Clayton URey.

Prof. Urey's first important piece of work consisted in carrying out extensive, accurate, spectroscopic measurements on diatomic and polyatomic molecules. This led him in 1931 to take up a detailed investigation on the abundance of natural isotopes of hydrogen, nitrogen and oxygen. During the next few years he succeeded in isolating deuterium and calculating the comparative thermodynamic properties of deuterium, hydrodeuterium and hydrogen. In 1934 he accomplished the first synthesis of deuteromethane.

Deuterium or 'labelled' hydrogen has proved of great value in investigating the mechanism of many organic and biologically important reactions, and its use has been the precursor of the modern general isotopic exchange reactions. A number of deutero derivatives have been prepared by Urey and his coworkers, and their entropies, vapour pressures and exchange equilibrium constants have been experimentally determined and compared with the the. oretical values anticipated.
This isolation of deuterium from ordinary hydrogen and establishment of the thermodynamic, spectral and physico-chemical difference between it and pure hydrogen, as well as in the compounds containing deuterium and hydrogen, is a remarkably complete piece of work, for which Urey received the Nobel Prize.

More recently Urey has taken up the problem of the separation of the other important, naturally occurring isotopes: those of nitrogen, oxygen and carbon. He has examined their quantity distribution in Nature and employed exchange methods for the enrichment of one species.

\section{DaRWIN Medal}

The Darwin Medal has been awarded to Prof. JAMES Peter Hill.

Over a long series of years Prof. Hill has carried out researches on the development of various mammals, particularly as regards the embryonic membranes and placenta, and added greatly to our knowledge of this subject. Many of his conclusions have clear evolutionary implications, as for example that marsupials are descended from oviparous ancestors with meroblastic ova. In his Croonian Lecture of 1932 Prof. Hill summarized his researches on the embryology and embryonic membranes of the Primates. The views of primate evolution based on development which he then put forward are in accord with those of Elliot Smith founded on brain anatomy, and of W. K. Gregory on morphological and palæontological evidence.

In collaboration with T. T. Flynn, Prof. Hill has lately (1939) published the first part of extensive researches on the development of monotremes, both Ornithorhynchus and Echidna, which will be of great value in helping to.assess the origin and relationships of these egg-laying mammals.

Prof. Hill's research work is of first-class quality, being trustworthy and carried out with extreme care and the best techniques; it has never been scamped and is rich in original results over a wide field, most of the conclusions having a direct bearing on evolutionary questions.

Few living biologists have contributed more towards the solution of problems bearing on the interrelationships of the main groups of the Mammalia and on the phylogenetic history of the Primates, a subject with which Charles Darwin was so much concerned.

\section{Sylvester Medal}

The Sylvester Medal has been awarded to Prof. Godfrey Harold Hardy.

G. H. Hardy is the author, or part author, of more than three hundred mathematical papers, two books, and several of the Cambridge Mathematical Tracts.

Much of his work has been directed to the building up of the technique of modern mathematical analysis, and the simplicity with which the routine aspects of new work can now be presented is due very largely to fundamental results established by him.

It is characteristic of much of his work that it has stimulated others and has proved to be the startingpoint of important developments. His work in collaboration with J. E. Littlewood on Tauberian theorems is an example. From an isolated classical result a subject was created which to-day would require a treatise for its exposition. 
His most outstanding contributions to the advance of mathematical knowledge have been in the theory of the Riemann zeta-function and the theory of numbers. The achievement of which, it is believed, he himself is most justly proud is the invention of the 'circle method'. This is a technique of much beauty and generality, which brings great refinement of mathematical analysis to bear on a wide class of unsolved problems in the theory of numbers. The method has been elaborated and improved by other mathematicians, but on its account alone the name of Hardy must for all time rank high among the masters of his subject.

No appreciation of the services of Hardy to the advance of mathematics would be complete which did not attempt to assess the value of his personal influence. Throughout his career he has been the driving force behind a vigorous group of younger research workers. A very considerable proportion of the pure mathematical research now being published in Great Britain is traceable more or less directly to his interest and encouragement, or to the inspiration of his earlier work. His unstinted service during many years to the detailed work of the London Mathematical Society, and the freedom with which his experience and advice are available to all, have established him in a unique position in the regard of British mathematicians.

\section{Hughes Medal}

The Hughes Medal has been awarded to Prof. Arthur Holly Compton.

Prof. Compton has made a number of important contributions to physical science in the field of $\mathrm{X}$-rays and elsewhere. Of late years he has been one of the leaders in the study of cosmic rays.

The experiments of Young and Fresnel early in the nineteenth century proved that light certainly had undulatory properties. But in the present century facts have been emerging, notably in con- nexion with photo-electric action, which are impossible to reconcile with the assumption that light can be described only as an electromagnetic wave of the classical type. These difficulties disappeared if light of frequency $v$ is assumed to be dynamically equivalent to a collection of particles of energy $h \nu(h$ is Planck's constant).

It occurred to Compton that from this point of view the interaction between radiation and free electrons is very simple, and in fact is the simplest interaction which radiation can undergo. Associated with the energy $h v$, according to the electromagnetic theory, there is momentum $h v / c$ ( $c$ is velocity of light). The interaction is thus reduced to a very ancient problem, that of the encounter of two infinitesimal billiard balls with known energies and moments. As the radiation moves with the velocity of light, in most cases the electron can be treated as if it were at rest. It is then obvious that in the collision the electron will acquire energy from the radiation, and the conservation of momentum requires that if the electron moves off in a certain direction the radiation will travel in a certain other direction. But reduction of energy of a quantum of radiation means increase in wave-length, and this increase will be a predetermined function of the direction of the 'scattered' radiation and of the direction of motion of the 'recoil' electron.

Compton published these conclusions in 1922. In 1923 he established the change in wave-length, first qualitatively by Barkla's absorption coefficient methods and then quantitatively with the X-ray spectrometer. In the succeeding years he investigated the energies of the recoil electrons as a function of their direction of motion and showed that the correlation, predicted by the theory, between the direction and energy of the recoil electrons on one hand and the direction and ehange of wave-length of the radiation on the other did in fact occur. This correlation is of fundamental importance in the general theory of the interaction of radiation with matter.

\section{BIOLOGICAL APPLICATIONS OF SYNTHETIC CHEMISTRY}

$\mathrm{P}^{\mathrm{n}}$ ROF. J. W. COOK, formerly at the Royal Cancer Hospital, who recently succeeded the late Prof. George Barger as regius professor of chemistry in the University of Glasgow, opened the winter session of the evening meetings in Edinburgh of the Pharmaceutical Society with a lecture on "Some Biological Applications of Synthetic Chemistry".

Prof. Cook said that many new facts have been recorded regarding the natures and the functions of substances which play vital parts in the process of life, and large numbers of these substances have been prepared artificially. Many rare compounds, not known in Nature, and yet possessing powerful biological activity, have arisen from the creative efforts of the synthetic chemist. The spectacular results which followed the introduction of the sulphonamide drugs were the outcome of purely chemical investigations. There is no doubt that prontosil, with its colourless prototype sulphanilamide, is destined to be regarded as one of the greatest boons to mankind conferred by this present age. Of the many thousands of sulphonamides that have now been prepared and examined, none surpasses sulphanilamide in efficacy against streptococcal infections. A later product, M. and B. 693, a sulphapyridine, has robbed pneumonia of much of its terror, and the analogous thiazole derivative has been claimed as effective against infections due to staphylococcus. These claims have not, however, been substantiated by tests carried out in Great Britain.

Much progress has also been made in other branches of chemotherapy; new products are constantly being evolved which have therapeutic and pharmacological properties resembling, and sometimes exceeding, those of natural plant products. Carefully planned researches, such as those of the Drug Addiction Committee of the United States Public Health Service, should receive every possible encouragement. The very rapid decline in the use of cocaine as an addiction drug, after the discovery of synthetic substitutes such as procaine, gave a stimulus to efforts to solve the problem of drug addiction with the opium 